\title{
Article
}

\section{Effect of Crystallization on Drawdown Force at Capillary Extrusion for Polyethylene}

\author{
Jiraporn SEEMORK ${ }^{*, * *}$, Tomoki ITOH ${ }^{*}$, Shogo NobUKAWA ${ }^{*}$, and Masayuki YAMAGUCHI ${ }^{* \dagger}$ \\ "School of Materials Science, Japan Advanced Institute of Science and Technology \\ 1-1 Asahidai, Nomi, Ishikawa 923-1292 JAPAN \\ ${ }^{* *}$ Program in Petrochemistry, Faculty of Science, Chulalongkorn University \\ Phayathai Rd., Bangkok 10330 THAILAND \\ (Received : September 29, 2015)
}

\begin{abstract}
The effect of crystallization on the drawdown force, i.e., the force needed to stretch a molten polymer uniaxially, was studied using conventional polyethylene samples. It was found that the drawdown force of high-density polyethylene (HDPE) increases with increasing the die length. This is attributed to the rapid crystallization owing to the low density of entanglement couplings after passing through a long die. On the contrary, the die length has no/little influence on the drawdown force of linear low-density polyethylene (LLDPE), indicating that the molecular orientation applied in a long die is almost relaxed prior to the crystallization. Even for LLDPE, however, the drawdown force becomes sensitive to the die length by the addition of a small amount of HDPE, because the crystallization process is accelerated.
\end{abstract}

Key Words: Elongational flow / Drawdown force / Polyethylene / Capillary extrusion / Crystallization

\section{INTRODUCTION}

Rheological responses of a molten polymer under elongational flow have received considerable attention in both academic and industrial fields, because they are important for polymer characterization and the quality of a product obtained by various processing operations in which a polymer melt with free surface is deformed, such as blow-molding, thermoforming, extrusion coating, foaming and spinning. ${ }^{1-3)}$ Therefore, the elongational viscosity has been studied greatly to predict the processability. The elongational viscosity is usually evaluated by commercially available rheometers such as Meissner-type rheometer ${ }^{3,4)}$ and Sentmanat extension rheometer (SER). ${ }^{5)}$ The measurements by the rheometers are, however, not so easy especially for a low viscous melt. Therefore, the drawdown force, defined as the force needed for uniaxial stretching of a polymer melt, is often evaluated in industry, instead of elongational viscosity. The drawdown force, which is also called melt strength or melt tension, can be evaluated even for a low viscous melt with a small experimental error. Furthermore, it has been believed that the drawdown force measured by various draw ratios has

Corresponding author

Tel: +81-761-51-1621, Fax: +81-761-51-1149, E-mail: m_yama@jaist.ac.jp equivalent information on the elongational viscosity. In fact, Bernnat reported that the growth curve of uniaxial elongational viscosity can be calculated by the drawdown force measurements. ${ }^{6}$ )

In our previous work, however, it was found that the drawdown force increases with the length of a die using isotactic polypropylene $(\mathrm{PP})^{7)}$, which is pronounced for a melt with a long relaxation time. Therefore, the drawdown force is sensitive to the die length at low temperature for a high molecular weight material. Furthermore, a stretched strand with a large drawdown force is found to have high level of molecular orientation. These results suggest that a melt passing through a long die has low density of entanglement couplings because the polymer chains disentangle and orient to the flow direction by the applied shear stress in the die. This situation becomes prominent beyond the shear strain at stress-overshooting. The reduced interaction of entanglement couplings leads to the rapid crystallization during stretching, owing to the enhanced molecular diffusion onto the surface of crystalline nuclei. In other words, polymer chains in a molten state do not have to "reptate" in the entanglement network to reach to the crystalline surface. This is similar to the flow-induced crystallization at injectionmolding. Such phenomenon has been reported at fiber spinning for nascent powders. ${ }^{8-11)}$ Furthermore, the prompt 
crystallization from liquid crystalline state is a well-known phenomenon as reported by Cheng et al. ${ }^{12)}$ Recently, the rapid crystallization from an ordered melt was also found for PP and applied to prepare a product with thick lamellae, i.e., high melting point. ${ }^{13)}$ The steep increase in the viscosity by the prompt crystallization is the origin of the drawdown force enhancement when using a long die.

This phenomenon has to be well understood for polyethylene (PE), because various types of PE with different molecular structure are commercially available. Further, their processability is often predicted by the drawdown force as well as melt flow rate (MFR). However, such researches have not been performed yet to the best of our knowledge, although the effect of short- and long-chain branches in PE on the elongational viscosity at a constant temperature has been widely studied. ${ }^{1-3,14-19)}$ According to the studies, long-chain branches are responsible for the strain-hardening behavior in the transient elongational viscosity owing to the chain stretching. Therefore, PE with long-chain branches shows high level of the drawdown force. In contrast, short-chain branches barely affect the elongational viscosity. Considering our previous result using PP, however, the crystallization behavior should affect the drawdown force of PE. The crystallization behavior is known to be strongly affected by the short-chain branches. For example, Gelfer and Winter revealed that the solidification rate depends on the branch content and the branching distribution but the latter has more strong influence. ${ }^{20)}$ In this study, the effect of short-chain branches on the drawdown force is evaluated considering the crystallization during stretching. For the purpose, three types of circular dies having different length with the same diameter are employed for the drawdown force measurements.

\section{EXPERIMENTAL}

\subsection{Materials and Sample Preparation}

Materials used in this study were commercially available high-density polyethylene (HDPE) and linear low-density polyethylene (LLDPE) having different molecular weights. Characteristics of the materials are summarized in Table I, in which the numeral in the sample code denotes the value of the melt flow rate (MFR) at $190{ }^{\circ} \mathrm{C}$. For example, HDPE-5 is the high-density polyethylene whose MFR is $5 \mathrm{~g} / 10 \mathrm{~min}$. HDPE-1, HDPE-5 and LLDPE-2 were produced by Ziegler-Natta catalyst, whereas LLDPE-1 was prepared by a metallocene catalyst. The crystallization temperature $T_{c}$, shown in Table I, was evaluated by a differential scanning calorimeter (DSC 820, Mettler-Toledo, USA). The samples were heated to $190^{\circ} \mathrm{C}$ at a heating rate of $30^{\circ} \mathrm{C} / \mathrm{min}$ to erase the thermal history, followed by the cooling process to $25{ }^{\circ} \mathrm{C}$ at a cooling rate of $10^{\circ} \mathrm{C} / \mathrm{min}$ to evaluate $T_{c}$. The number of short-chain branches per 1000 backbone carbon atoms were characterized by carbon nuclear magnetic resonance spectroscopy $\left({ }^{13} \mathrm{C}\right.$-NMR). LLDPE-1 is a copolymer of ethylene and 1-hexene, while LLDPE-2 is that of ethylene and 1-butene. The molecular weight and its distribution were evaluated by a size exclusion chromatography as polyethylene standard.

Besides pure PE samples, two blends were prepared by the addition of $1 \mathrm{wt} \%$ of HDPE- 5 into either LLDPE- 1 or LLDPE-2 using an internal batch mixer (Toyoseiki, LaboPlastmill, Japan). A blade rotation speed, temperature and mixing time were $30 \mathrm{rpm}, 160{ }^{\circ} \mathrm{C}$ and $3 \mathrm{~min}$, respectively. During melt mixing, $5000 \mathrm{ppm}$ of a thermal stabilizer, 6-[3-(3-t-butyl-4-hydroxy-5-methylphenyl)propoxy]-2,4,8,10tetra-t-butyldibenzo [d,f] $[1,3,2]$ dioxaphosphepin (Sumilizer ${ }^{\circledR}$ GP, Sumitomo Chemical, Japan), and 1000 ppm of calcium stearate were added. The samples obtained were compressed into a flat sheet at $200^{\circ} \mathrm{C}$ for 3 min prior to the measurements.

\subsection{Measurements}

The frequency dependence of oscillatory shear moduli such as storage modulus $G^{\prime}$ and loss modulus $G^{\prime \prime}$ was obtained by a cone-and-plate rheometer (AR2000, TA Instruments, USA) at $190{ }^{\circ} \mathrm{C}$. The diameter and the cone angle were $20 \mathrm{~mm}$ and 5 degree, respectively.

The drawdown force was measured by a capillary rheometer (140 SAS-2002, Yasuda Seiki Seisakusyo, Japan) equipped with a set of stretching wheels and a tension detector. The experiment was performed at a shear rate of $21.4 \mathrm{~s}^{-1}$ using various circular dies with $1 \mathrm{~mm}$ diameter. The

Table I. Characteristics of the materials.

\begin{tabular}{|c|c|c|c|c|c|c|}
\hline Sample & $\begin{array}{c}\text { MFR } \\
(\mathbf{g} / \mathbf{1 0} \mathbf{~ m i n})^{1}\end{array}$ & $\begin{array}{c}\text { Number of } \\
\mathbf{S C B}^{2}\end{array}$ & $\mathbf{M n}$ & $\mathbf{M w}$ & $\begin{array}{c}\boldsymbol{\eta}_{\mathbf{0}} \\
\left(\mathbf{P a ~ s}^{\mathbf{3}}\right.\end{array}$ & $\begin{array}{c}\boldsymbol{T}_{\boldsymbol{c}} \\
\left.\mathbf{(}^{\circ} \mathbf{C}\right)^{4}\end{array}$ \\
\hline \hline HDPE-1 & 0.95 & - & $1.7 \times 10^{4}$ & $1.0 \times 10^{5}$ & $1.8 \times 10^{4}$ & 116.4 \\
\hline HDPE-5 & 5.0 & - & $1.2 \times 10^{4}$ & $7.5 \times 10^{4}$ & $2.2 \times 10^{3}$ & 115.7 \\
\hline LLDPE-1 & 1.0 & $\begin{array}{c}18.4 \\
(\text { Butyl })\end{array}$ & $3.8 \times 10^{4}$ & $1.0 \times 10^{5}$ & $8.0 \times 10^{3}$ & 100.1 \\
\hline LLDPE-2 & 2.0 & $\begin{array}{c}18.0 \\
(\text { Ethyl })\end{array}$ & $2.6 \times 10^{4}$ & $1.1 \times 10^{5}$ & $7.7 \times 10^{3}$ & 104.6 \\
\hline
\end{tabular}

${ }^{1}$ Melt flow rate at $190{ }^{\circ} \mathrm{C}$ and $2.16 \mathrm{kgf}$

${ }^{2}$ Number of short-chain branches per 1000 backbone carbons

${ }^{3}$ Evaluated at $190{ }^{\circ} \mathrm{C}$

${ }^{4}$ Measured by DSC at $-10{ }^{\circ} \mathrm{C} / \mathrm{min}$ 
length-to-diameter ratios $(L / D)$ of the dies were $10 / 1,20 / 1$ and 40/1. Temperature in a capillary reservoir and the die was controlled at $190{ }^{\circ} \mathrm{C}$. The sample was extruded from the die at a constant volume rate and stretched at a draw ratio of 4.6 to measure the drawdown force. The diameter of a molten strand was also measured by a laser beam without stretching to calculate the swell ratio.

\section{RESULTS AND DISCUSSION}

Fig. 1 shows the oscillatory shear moduli for the materials used in this study. As seen in the figure, the order in loss modulus corresponds with MFR. In the case of HDPE-1, the storage moduli in the low frequency region are relatively high, suggesting that it has broad molecular weight distribution. Furthermore, the storage moduli in the low frequency region for LLDPE-2 is slightly higher than those for LLDPE-1. This is also attributed to the difference in the molecular weight distribution as shown in Table I. Since LLDPE-1 is produced by a metallocene catalyst, it has narrow molecular weight distribution. Moreover, the data of zero-shear viscosity are also summarized in the table to confirm the difference in the molecular weight.

The values of drawdown force evaluated using various dies with different length are shown in Fig. 2. As seen in the data of the LLDPE samples, the molecular weight distribution does not affect the drawdown force significantly. Furthermore, it should be noted that the drawdown force increases with increasing the die length for HDPE, which is a similar phenomenon detected using PP. ${ }^{7)}$ The applied flow history in a long die reduces the density of entanglement couplings, because the polymer chains will disentangle and orient to the flow direction by the strong shear stress in the die. As a result, chain diffusion onto the crystal surface is greatly enhanced during the crystallization process under stretching, as explained in detail in the introduction. This is responsible for the prompt crystallization and the high level of drawdown force, which was described in our previous work. ${ }^{7)}$ In the case of LLDPE, in contrast, the drawdown force values are not sensitive to the die length, i.e., it does not increase with increasing the die length. In our previous study using PP, it was revealed that the drawdown force is not sensitive to the die length at high extrusion temperature. ${ }^{7)}$ This result indicates that the die length sensitivity can be explained by the difference in $\Delta T$ (processing temperature $-T_{c}$ ) and crystallization rate. Considering that the cooling rate of an extrudate becomes significantly slow at low temperature due to the small temperature difference between circumstance and the sample, LLDPE requires a long solidification time due to the low $T_{c}$. Therefore, the steep increase in the viscosity during cooling is not expected for LLDPE because of the slow crystallization rate and the low degree of crystallinity as compared with HDPE. As a result, the chain orientation applied in the long die is relaxed greatly prior to the crystallization for LLDPE. Once the chains are relaxed, i.e., recoiled, the crystallization takes place more slowly, leading to no/little enhancement of the drawdown force and then less sensitivity to the die length.

In the case of HDPE-5, the drawdown force is almost the same as those of the LLDPE samples when using the long die, even though the shear viscosity of HDPE-5 is significantly lower than those of the LLDPE samples. It indicates that
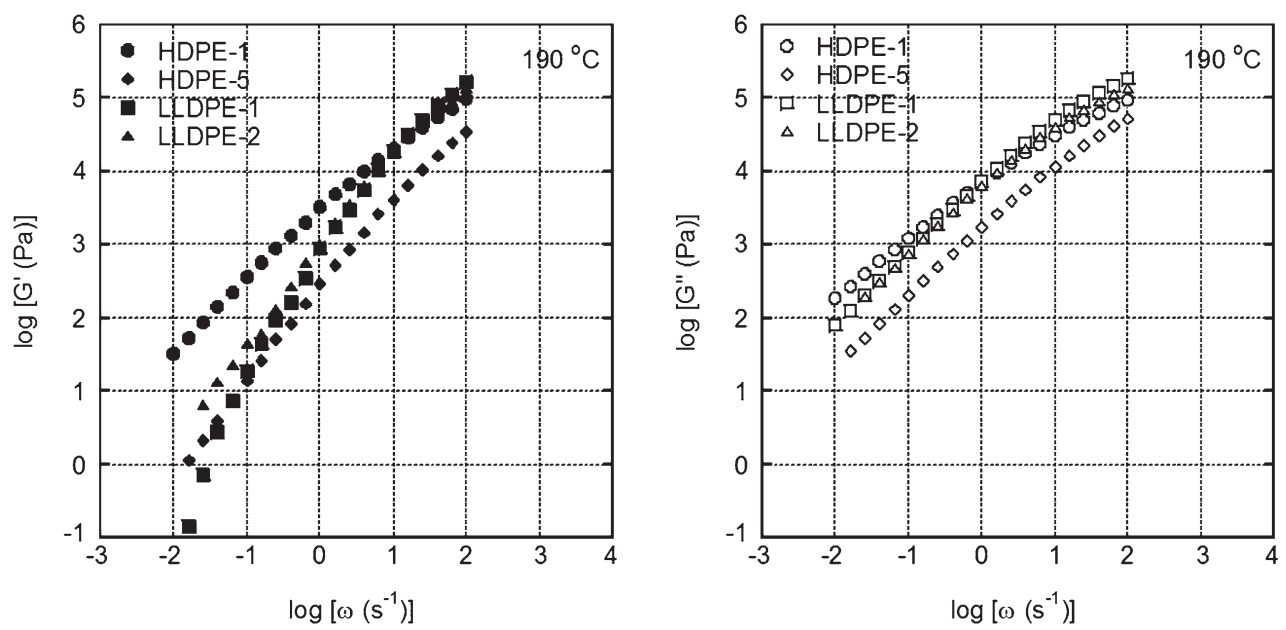

Fig. 1. Angular frequency dependence of oscillatory shear moduli such as storage modulus $G^{\prime}$ (left) and loss modulus $G^{\prime \prime}$ (right) for HDPE-1 (circles), HDPE-5 (diamonds), LLDPE-1 (squares) and LLDPE-2 (triangles) at $190^{\circ} \mathrm{C}$. 
HDPE-5 shows prompt crystallization with a large amount of crystallinity as compared with LLDPE, resulting in the high level of the drawdown force. Moreover, the results demonstrate that a great attention has to be paid to compare the drawdown force of PE samples having different $T_{c}$ and/or crystallinity.

The reduction of entanglement couplings in a long die also affects the extrudate swell, which was intensively studied by Tanner. ${ }^{21)}$ According to him, the swell ratio of a molten polymer is determined by two factors; one is the primary normal stress difference and the other is the memory effect. The difference in die length affects the latter mechanism because the entanglement density in a field at a low shear rate, e.g., in a cylinder of a capillary rheometer, is higher than that in a high shear rate area, e.g., in a die.

As shown in Fig. 3, the swell ratio decreases with increasing the die length for HDPE-1 and LLDPE-1 with an intense fashion of HDPE-1. The result demonstrates that the swell ratio of a melt with a long relaxation time is sensitive to the die length. Furthermore, it is barely affected by $T_{c}$ of the samples. This is reasonable because the enlargement of strand diameter, i.e., extrudate swell, occurs immediately after the die exit, i.e., prior to the crystallization.

As mentioned, the drawdown force is often measured to predict the elongational viscosity. Besides, the current study reveals that the drawdown force contains the information on crystallization process, which is affected by the flow history in a die and prominent for a polymer with high $T_{c}$ and crystallinity. The latter information is, of course, also important to predict the processability at various processing

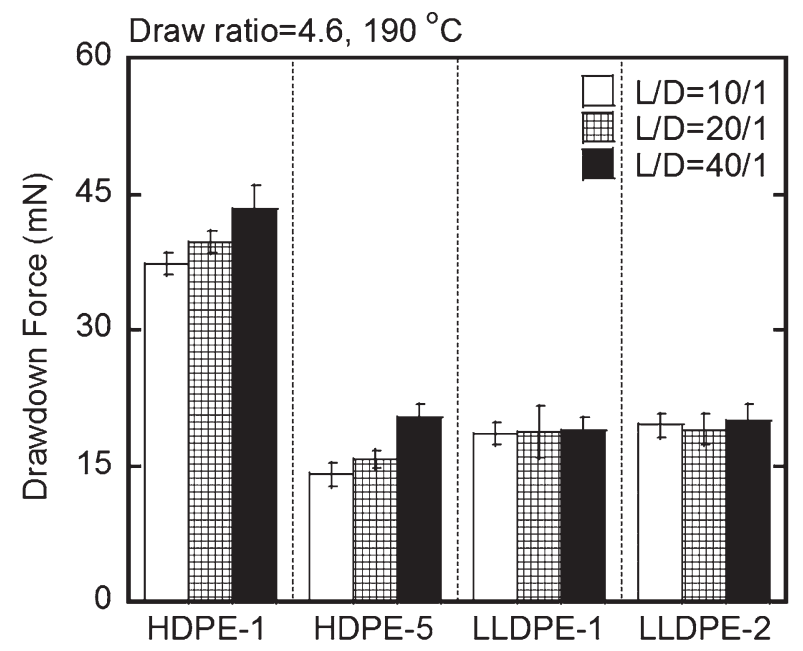

Fig.2. Drawdown force evaluated using various dies at a draw ratio of 4.6 for HDPE-1, HDPE-5, LLDPE-1 and LLDPE-2. The extrusion was carried out at $190{ }^{\circ} \mathrm{C}$. operations, such as stability of a tubular-blown film, neck-in at T-die extrusion and expansion ratio at foaming. To confirm the role of crystallization in the drawdown force, we performed further experiments using the LLDPE blends containing $1 \mathrm{wt} \%$ of HDPE-5. As shown in Fig. 4, the drawdown force values of both LLDPE samples become sensitive to the die length by the HDPE addition, i.e., increase with increasing the die length, which is significantly different from the trend of the neat LLDPE. As previously reported, cocrystallization takes place in PE blend systems, e.g., LLDPE/HDPE blends. ${ }^{22,23)}$ As a result, the HDPE addition enhances $T_{c}$ and crystallization rate of LLDPE. A similar phenomenon was

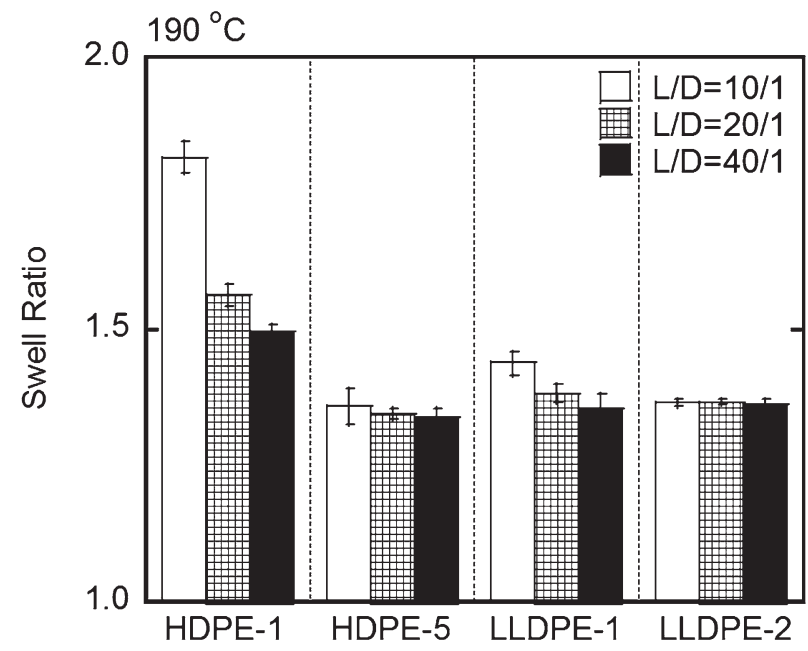

Fig.3. Swell ratio of HDPE-1, HDPE-5, LLDPE-1 and LLDPE-2 evaluated using various dies at $190{ }^{\circ} \mathrm{C}$. The apparent shear rate at die wall is $21.4 \mathrm{~s}^{-1}$.

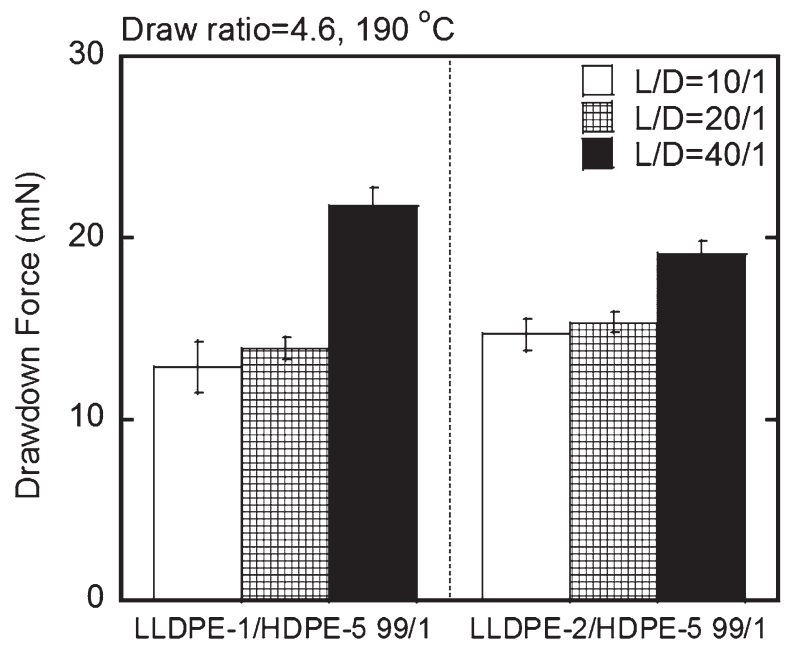

Fig.4. Drawdown force of the blends evaluated using various dies at a draw ratio of 4.6; (left) LLDPE-1/HDPE-5 (99/1) and (right) LLDPE-2/ HDPE-5 (99/1). The extrusion was carried out at $190^{\circ} \mathrm{C}$. 
observed when a small amount of LDPE is added to LLDPE containing a large amount of short-chain branches. ${ }^{24)}$ This is attributed to the nucleating ability of LDPE-rich crystals for the rest of LLDPE. As similar to these reports, the HDPE-rich crystals act as the nucleating agent also in this system, which accelerate crystallization process for the rest of LLDPE. Then, LLDPE shows rapid crystallization at relatively high temperature. Once the crystallization takes place, chain orientation applied in a long die contributes the prompt crystallization due to the marked diffusion ability onto the HDPE-rich crystals. Consequently, the drawdown force becomes sensitive to the die length even for LLDPE.

\section{CONCLUSION}

The effect of die length on the drawdown force is studied using various types of PE. It is found that the drawdown force of HDPE is enhanced when using a long die. The reduction of entanglement couplings in a long capillary die will be responsible for the prompt crystallization. The steep increase in the viscosity by the crystallization leads to the increase in the drawdown force. In the case of LLDPE, however, the die length barely affects the drawdown force, because the orientation applied in a long die will be relaxed prior to the crystallization. After the addition of a small amount of HDPE, the drawdown force of LLDPE increases with increasing the die length. The experimental results obtained in this study demonstrate that the drawdown force includes the information on not only elongational viscosity but also crystallization behavior. Furthermore, the die length affects the extrudate swell especially for a polymer with a long relaxation time regardless of the crystallization temperature.

\section{ACKNOWLEDGMENT}

The authors appreciate Prime Polymer Co., Ltd. for the characterization of the samples.

\section{REFERENCES}

1) Meissner J, Raible T, Stephenson SE, J Rheol, 25, 1 (1981).

2) Yamaguchi M, Takahashi M, Polymer, 42, 8663 (2001).

3) Koyama K, Nihon Reoroji Gakkaishi, 34, 267 (2006).

4) Meissner J, Hostettler A, Rheol Acta, 33, 1 (1994).

5) Sentmanat M, Rheol Acta, 43, 657 (2004).

6) Bernnat A, Doctor thesis, Univ Stuttgart, 2001.

7) Seemork J, Siriprumpoonthum M, Lee Y, Nobukawa S, Yamaguchi M, Adv Polym Technol, 34, 21477 (2015).

8) Kanamoto T, Ohama T, Tanaka K, Takeda M, Porter RS, Polymer, 28, 1517 (1987).

9) Sawai D, Miyamoto M, Kanamoto T, Ito M, J Polym Sci Polym Phys, 38, 2571 (2000).

10) Sano A, Iwanami K, Matsuura $S$, Yokoyama $S$, Kanamoto $T$, Polymer, 42, 5859 (2001).

11) Sawai $\mathrm{D}$, Watanabe $\mathrm{D}$, Morooka $\mathrm{N}$, Kuroki $\mathrm{H}$, Kanamoto $\mathrm{T}, J$ Polym Sci Polym Phys, 44, 3369 (2006).

12) Pardey R, Wu SS, Chen J, Harris FW, Cheng SZD, Keller A, Aducci J, Facinelli JV, Lenz RW, Macromolecules, 27, 5794 (1989).

13) Phulkerd $P$, Arayachukeat $S$, Huang $T$, Inoue $T$, Nobukawa $S$, Yamaguchi M, J Macromol Sci B, 53, 1222 (1989).

14) Münstedt H, Kurzbeck S, Egersdörfer L, Rheol Acta, 37, 21 (1998).

15) Yamaguchi M, Wagner MH, Polymer, 47, 3629 (2006).

16) Sugimoto $M$, Masubuchi $Y$, Takimoto J, Koyama K, Macromolecules, 34, 6056 (2001).

17) Ajji A, Sammut P, Huneault MA, J Appl Polym Sci, 88, 3070 (2003).

18) Kim J, Kim DH, Son Y, Polymer, 50, 4998 (2009).

19) Mieda N, Yamaguchi M, J Non-Newton Fluid Mech, 166, 231 (2011).

20) Gelfer Y, Winter HH, Macromolecules, 32, 8974 (1999).

21) Tanner RI, in Rheological Measurement, Collyer AA, Clegg DW, Eds, Elsevier, London, 1988.

22) Hu SR, Kyu T, Stein RS, J Polym Sci Polym Phys, 25, 71-87 (1987).

23) Tashiro K, Izuchi M, Kobayashi M, Stein RS, Macromolecules, 27, 1221-1227 (1994).

24) Yamaguchi M, Abe S, J Appl Polym Sci, 74, 3153 (1999). 